\title{
A CASE REPORT OF TUMORAL CALCINOSIS
}

B.V. Sreedevi ${ }^{1}$,

\section{HOW TO CITE THIS ARTICLE:}

B.V. Sreedevi. "A Case Report of Tumoral Calcinosis". Journal of Evolution of Medical and Dental Sciences 2014; Vol. 3, Issue 07, February 17; Page: 1619-1624, DOI: 10.14260/jemds/2014/2034

ABSTRACT: Tumoral Calcinosis also called Tumoral Lipocalcinosis ${ }^{1}$ in which there is large calcareous swellings in the subcutaneous tissue near the larger joints e.g. elbow, shoulder, hips and knee. This condition may be familial and is seen most often in black Africans. It may be precipitated by minor trauma but its pathogenesis is obscure. In this case report, tumoral calcinosis was present in a female aged 70, as a recurrent chronic inflammatory swelling over a scar area with a discharging sinus.

KEYWORDS: Tumoral calcinosis, Dystrophic Calcification, Metastatic Calcification, Ossification, CREST Syndrome. Consent was obtained from the patient and his son.

CASE REPORT: A 70 year old female, laborer by occupation coming from Ramnad Village presented with complaints of recurrent chronic painful swelling on the upper lateral aspect of right thigh for two months. Patient gives history of developing a small nodular lesion on the lateral aspect of right thigh which was operated by a practitioner in the village. One month later she developed a small swelling which slowly grew in size to reach the present size. For the past two months, she has developed a sinus in the swelling which started discharging sero purulent material. There was no history of trauma or any other significant medical illness. Patient was not a diabetic, hypertensive or a thyroid patient and was not suffering from any other skin ailment or bleeding disorders. She was not taking drugs for any other ailment. On Examination: Patient was thin built, vital signs were stable and all systems were normal. On local Examination: A swelling of 9x5 cm was seen on the upper lateral aspect of right thigh just below ASIS. The skin over the swelling was black in color with a sieve like opening which was discharging pus.

The swelling was tender, but was not mobile. On Investigation: Complete blood count was normal. Liver function test, renal function test and, urine examinations were normal. Work up for HIV was normal. FNAC showed chronic inflammatory cells. Discharge was sent for culture sensitivity which showed staphylococcus aureus grown in culture, sensitive to cephalosporin. X ray pelvis was taken. It showed a focus of calcification in the soft tissue adjacent to right anterior superior iliac spine. 64 slice CT scan pelvis was taken. Impression: Ill-defined heavily calcified lesion measuring $7.42 \times 2.33 \times 6.13 \mathrm{cms}$ was seen in the subcutaneous soft tissues in the right iliac fossa lesion overlying the right iliac crest and the anterior part of the right gluteus maximus muscle. There was no abnormal enhancement of the post contrast scans. Imaging features were suggestive of? hemangioma with phleboliths, ?chronic calcified abscess / hematoma. Management: The patient was treated with wide local excision and sent for HPE. PO period was uneventful and the Patient was reviewed 10 days later, one month later. No recurrence was observed. Histopathology report: Specimen: Right thigh swelling (wide local excision). Gross: Received soft tissue with attached strip of skin measuring 9.5X6X3.5 cm, $\mathrm{C} / \mathrm{s}$ - reveals multiple areas of calcification? Calcinosis, one area reveals irregular cyst with shaggy borders? Abscess measuring 1.5X1 cm. Microscopy: Section studied shows features consistent with calcinosis with associated inflammatory reaction. 


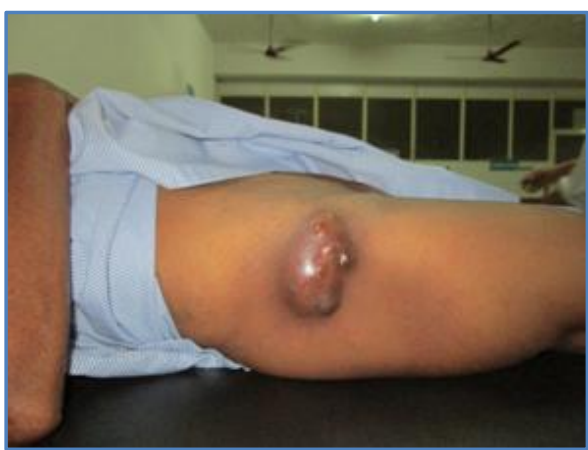

Picture showing patient presenting as chronic inflammatory swelling with discharging sinus.

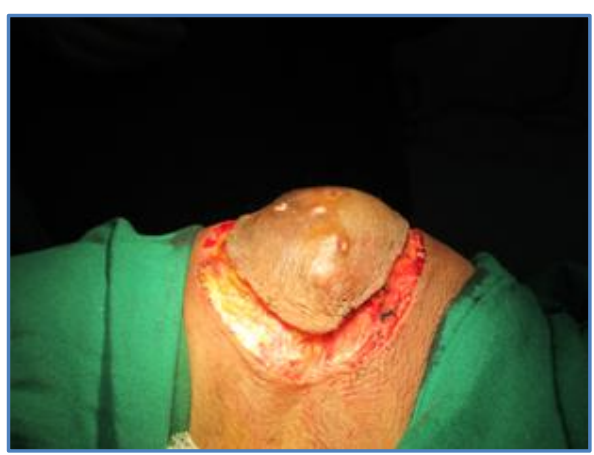

Patient undergoing wide surgical excision for Tumoral calcinosis.

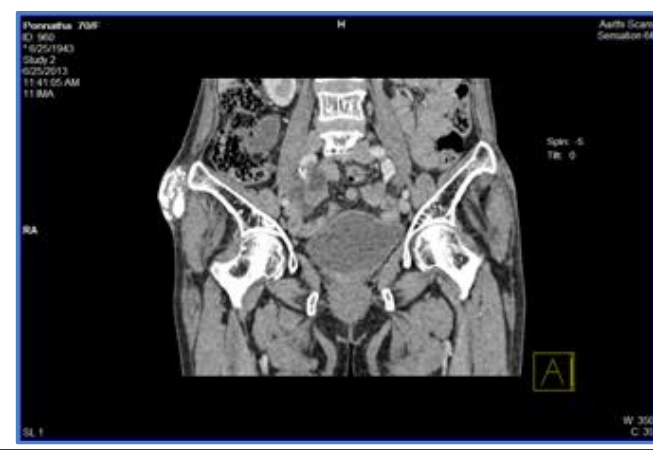

Picture of CT Scan PELVIS of the patient

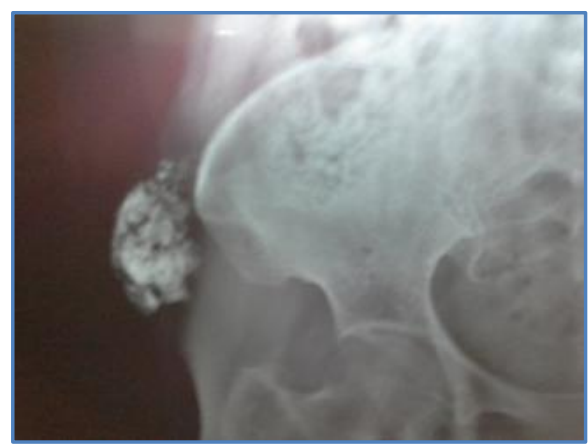

Picture of X Ray PELVIS of the patient

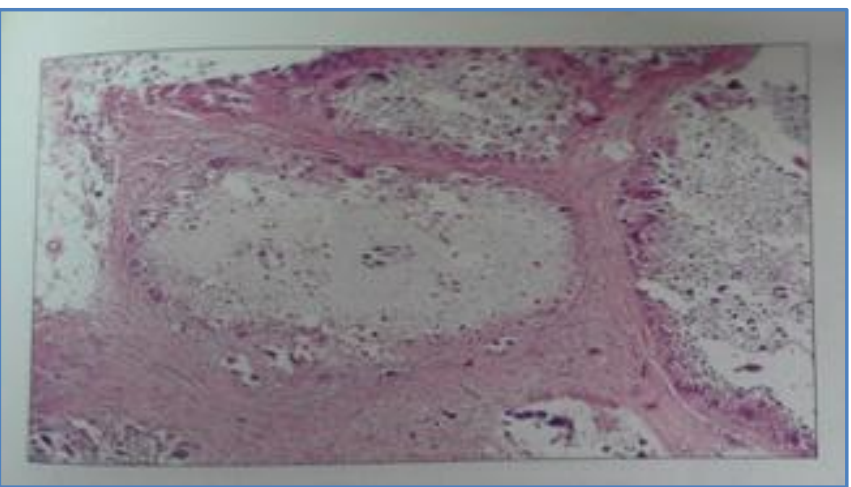

Tumoral calcinosis slide shows Amorphous calcified material bordered by a florid proliferation of macrophages multinodulated, osteoclast - like giant cells and are separated by bands of fibrousissue. 


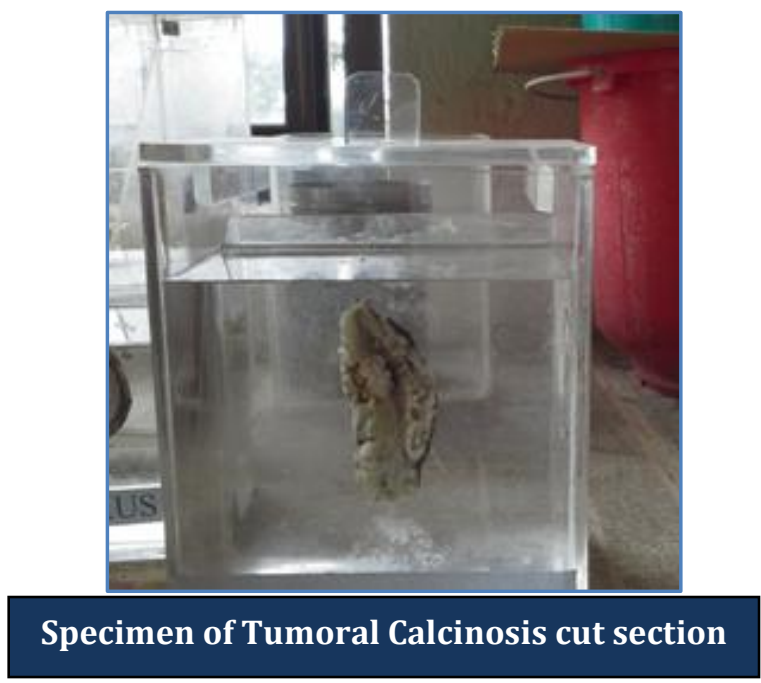

INTRODUCTION: Calcinosis is the formation of calcium deposits in any soft issue. Deposition ${ }^{2}$ of calcium phosphate crystals (calcification) or formation of true bone (ossification) in non-osseous soft tissue may occur by one of the three mechanisms:

1) Metastatic calcification due to supra normal calcium $X$ Phosphate concentration product in extra cellular fluid

2) Dystrophic calcification due to mineral deposition into metabolically impaired or dead tissue despite normal serum levels of calcium and phosphate

3) Ectopic ossification, or true bone formation. A constellation of calcinosis, Raynaud's phenomenon, esophageal dysmotility, Sclerodactyly and telangiectasia is called as CREST syndrome.

DISCUSSION: Back Ground: Calcinosis is the formation of calcium deposits in any soft tissue Virchow initially described calcinosis in 1855. Different types of calcinosis are

1) Calcinosis Cutis
a) Dystrophic Calcinosis Cutis
b) Metastatic Calcinosis Cutis
c) Iatrogenic Calcinosis Cutis
d) Traumatic Calcinosis Cutis
e) Idiopathic Calcinosis Cutis

2) Idiopathic Scrotal Calcinosis

3) Sub epidermal Calcified nodule

4) Tumoral Calcinosis

5) Calcinosis Cutis Circum Scripta

6) Calcinosis Cutis Universalis

7) Transplant associated calcinosis cutis.

TUMORAL CALCINOSIS: Definition: It is a distinct clinical and histologic entity that is characterized by tumor like peri articular deposits of calcium that are found mostly in the regions of hip, shoulder, and elbow. The disorder occurs predominantly in otherwise healthy children, adolescents and young 
adults. It is more often multiple than solitary and not infrequently affects 2 or 3 or more siblings of the same family.

HISTORY: The term tumoral calcinosis was coined by Inclan ${ }^{3}$ in 1943 but this condition was recognized as an entity much earlier. In $1899^{3}$ Duret observed this process in siblings. A 17 year old girl and her younger brother in the neighborhood had multiple calcifications of hip and elbow joint. Later in 1935, Teutschlaender gave a detailed account of another typical case of an 11 year old girl with multiple lesions in shoulder and elbow regions which had their onset at age of 2 years. He thought that this process was secondary to fat necrosis and used the term "Lipid Calcinosis" since then, numerous other acceptable examples of this growth have been reported under various names, including calcifying bursitis, calcifying collagenolysis and kikuyu bursa. In New guinea, the natives aptly refer to it as "hip stones"3.

Pathogenesis: Tumoral calcinosis is an inborn error in calcium metabolism which in most cases appear to be inherited as Autosomal Recessive pattern as studies reveal normal calcium levels but in many cases there is elevation of serum phosphates levels probably, the result of increased tubular reabsorption or reduced renal excretion of phosphorus. Subsequent studies over the past several years have implicated the GALNT3GENE located on 2924-31 which encodes for a Glycosyl Transferase responsible for initiating mucin type 0-glycosylation. The FGF23 gene which encodes for a potent phosphaturic protein has also been implicated as central to the pathogenesis of this disease. Trauma is rarely reported by patients with tumoral calcinosis but minor repeated trauma and tissue injury seem to play a role in calcifying process. It acts as a trigger mechanism in genetically susceptible individuals that leads to a chain of events, beginning with hemorrhage, fat necrosis, fibrosis and collagenisation and ending with collagenolysis and ultimately massive calcification.

Age: Usually occurs in first and second decade of life. It is rare in patients of older than 50 years. Sex: Overall male and female ratio is equal.

\begin{tabular}{|c|c|c|}
\hline Site & No of cases & $\%$ \\
\hline Hips & 33 & 31 \\
\hline Buttocks & 27 & 26 \\
\hline Upper extremities & 16 & 15 \\
\hline Lower extremities & 12 & 11 \\
\hline Spine / Sacrum & 7 & 7 \\
\hline Miscellaneous & 10 & 10 \\
\hline Total & 105 & 100 \\
\hline
\end{tabular}

Pathologic findings: Macroscopic-Study of the gross specimen discloses a firm rubbery mass that is unencapsulated extending to the adjacent muscles and tendons and is usually $5-15 \mathrm{~cm}$ in greater diameter. On Sectioning, the mass consists of a frame work of dense fibrous tissue containing spaces filled with yellow grey, pasty, calcareous material or chalky, milky liquid that is easily washed out, resulting in irregular cystic cavities. Chemical analysis of the intra and extra cellular calcified material 
reveals hydroxyapatite. Microscopically, active and inactive phases of the disease can be distinguished in the same lesion.

Clinical Features: The principal manifestation of the disease is the presence of a large, firm, subcutaneous calcified mass that is asymptomatic and slowly growing, often gradually enlarging over many years. It is usually located in the vicinity of a large joint especially the trochanteric and gluteal regions of the hip, the lateral portion of shoulder and posterior elbow. It is less frequent in hand, feet and knees. However the tumoral calcinosis has been described in many sites, including the spine, tempero mandibular joint and skin. The lesion is firmly attached to the underlying fascia, muscles or tendon and may infiltrate these structures but it is unrelated to bone. Approximately 2/3 of patients have multiple lesions, some of which are bilateral and symmetric. The underlying joints are unaffected, and with few exceptions patients with tumoral calcinosis are in good health.

Complications: Of this disease is rare, occasionally, there is ulceration of the overlying skin with secondary infection fistula formation, and discharge of a yellow white chalky fluid. Larger lesions may also affect nearby joints or impinge on anatomic structures. For example lesions arising near the wrist can cause carpal tunnel syndrome.

Investigations: Laboratory examinations show no evidence of increased calcium levels, but in many patients there is slight to moderate hypophosphatemia. Calcitriol may also be elevated, but serum alkaline phosphatase and uric acid levels are normal.

Examination with radiography, CTSCAN, or MRI reveals a subcutaneous conglomerate of multiple rounded opacities separated by radiolucent levels (Fibrous septa) imparting a "Chickenwire" pattern of lucencies with distinct fluid levels in some of the nodules. There are no associated bony abnormalities: despite the large amounts of calcium in the lesion, there is no evidence of osteoporosis in the skeleton.

\section{Differential Diagnosis:}

Tumor like calcific lesions in soft tissue can occur in

1) Tumoral calcinosis

2) Chronic renal failure with secondary hyper parathyroidism

3) Milk - Alkali Syndrome

4) Hypervitaminosis D

5) Bone destruction secondary to infections / neoplasm

Treatment: The treatment of choice is surgical removal of lesion as early as possible when the lesion is still small and amenable to total resection incomplete excision may lead to recurrence, secondary infection abscess formation. A number of other medical therapies have been attempted but with limited success.

\section{Other types of calcinosis:}

1) Calcinosis cutis - Calcinosis cutis ${ }^{4}$ is a type of calcinosis where calcium is deposited in the skin. 
2) Calcinosis circumscripta - Calcium deposits in subcutis chiefly affects middle aged women and most commonly involves the hand and wrist including tendon sheath. It is associated in large percentage of cases with Reynaud's disease or scleroderma, sclerodactyly or polymyositis.

3) Calcinosis universalis where calcium is deposited in skin and this forms multiple nodules or plaques that occur mainly in children and are associated in about half of the cases with manifestations of scleroderma or dermato myositis. It may ultimately lead to limited mobility, contractures and ankylosis.

CONCLUSION: This 70 year old female, with no family history and with no other clinical biochemical, radiological abnormality, presented with recurrence and in the scar area as a chronic inflammatory swelling with a discharging sinus which was treated successfully with wide local excision with no recurrence.

\section{ACKNOWLEDGEMENT:}

1) HOD, Department of General Surgery, Tagore Medical College \& Hospital

2) HOD, Department of Anesthesiology, Tagore Medical College \& Hospital

3) HOD, Department of Pathology, Tagore Medical College \& Hospital

\section{REFERENCES:}

1. J.B. Walter I.C. Talbot, Walter and Israel General Pathology, $7^{\text {th }}$ Ed, 2009 Elsevier. Churchill Livingstone. P- 647.

2. Dan L Longo, Dennis L Kasper, J Larry Jameson, Anthonys S Fauci, Stephen L Hauser, Joseph Loscalzo, Harrison's Principles of Internal Medicine. 18 $8^{\text {th }}$ Ed Volume II 2012. Newyork McGraw Hill Company P-3143.

3. Sharon W Weiss, John R Gold blum, Enzinger and Weiss. Soft Tissue Tumour, 5th Ed 2008. Mosby, Inc, Elsevier P-1063-67.

4. David A Warell, Timothy M Cox, John D Firth, Oxford Textbook of Medicine, $5^{\text {th }}$ Ed Vol 2 Sect 13-19. Oxford, Oxford University Press, 2010 P-3694.

\section{AUTHORS:}

1. B.V. Sreedevi.

\section{PARTICULARS OF CONTRIBUTORS:}

1. Associate Professor, Department of General Surgery, Tagore Medical College and Hospital, Rathinamangalam, Kanchipuram Dist., Tamilnadu.

\section{NAME ADDRESS EMAIL ID OF THE} CORRESPONDING AUTHOR:

Dr. B. V. Sreedevi, \#3, Gnanambal Garden, II ST, Ayanavaram, Chennai - 23.

E-mail: surgeonsreedevi@gmail.com

Date of Submission: 23/01/2014. Date of Peer Review: 24/01/2014. Date of Acceptance: 03/02/2014. Date of Publishing: 12/02/2014. 